Bond University

Research Repository

\title{
Does premenstrual syndrome before pregnancy increase the risk of postpartum depression? Findings from the Australian Longitudinal Study on Women's Health
}

Cao, Sifan; Jones, Mark; Tooth, Leigh; Mishra, Gita

Published in:

Journal of Affective Disorders

DOI:

10.1016/j.jad.2020.09.130

Licence:

CC BY-NC-ND

Link to output in Bond University research repository.

Recommended citation(APA):

Cao, S., Jones, M., Tooth, L., \& Mishra, G. (2021). Does premenstrual syndrome before pregnancy increase the risk of postpartum depression? Findings from the Australian Longitudinal Study on Women's Health. Journal of Affective Disorders, 279, 143-148. https://doi.org/10.1016/j.jad.2020.09.130

\section{General rights}

Copyright and moral rights for the publications made accessible in the public portal are retained by the authors and/or other copyright owners and it is a condition of accessing publications that users recognise and abide by the legal requirements associated with these rights.

For more information, or if you believe that this document breaches copyright, please contact the Bond University research repository coordinator 
Does premenstrual syndrome before pregnancy increase the risk of postpartum depression? Findings from the Australian Longitudinal Study on Women's Health

Authors: Sifan Cao, MMed ${ }^{1 *}$; Mark Jones, $\mathrm{PhD}^{2}$; Leigh Tooth, $\mathrm{PhD}^{1}$; Gita Mishra, $\mathrm{PhD}^{1}$

${ }^{1}$ Faculty of Medicine, School of Public Health, Centre for Longitudinal and Life Course Research, The University of Queensland, Brisbane, Queensland, Australia

${ }^{2}$ Faculty of Health Sciences \& Medicine, Bond University, Gold Coast, Queensland, Australia

\section{$\underline{\text { Abstract }}$}

\section{Background}

Previous literature suggests a positive association between history of premenstrual syndrome (PMS) and development of postpartum depression (PPD); however, limited evidence has come from prospective population-based studies and whether history of depression affects this association is unknown.

\section{Methods}

This study included 5479 women from the 1973-78 cohort of the Australian Longitudinal Study on Women's Health and estimated the association between pre-pregnancy PMS and PPD. Participants were followed from 22-27 years in 2000 to 37-42 years in 2015. PMS was collected from a 4category Likert-scale reporting on frequency of PMS in the last 12 months (never, rarely, sometimes, or often) at the survey preceding an index birth. PPD was ascertained from reports of doctor diagnoses for each birth. Relative risks (RRs) and 95\% confidence intervals (CIs) were used to estimate the association of interest. The role of history of depression was assessed by testing its interaction with pre-pregnancy PMS.

\section{Results}

During 15 years' follow-up, 15.4\% of participating women reported PPD; and 55.1\% reported PMS (rarely: $17.2 \%$, sometimes: $25.7 \%$, and often: $12.2 \%$ ). Compared to women who had no PMS

\footnotetext{
* Corresponding author: Sifan Cao, 288 Herston Rd, Herston QLD 4006, Australia, sifan.cao@uq.edu.au
} 
before pregnancy, those who rarely had PMS had similar risk of PPD (1.03, 0.82-1.30); whereas those who sometimes or often had PMS had significantly higher risk of PPD (1.31, 1.09-1.57 and $1.51,1.22-1.87$, respectively). History of depression did not affect the association.

\section{Limitations}

PMS was self-reported. PMS severity was not collected.

\section{Conclusions}

This large population-based study provides evidence of a dose-response relationship between PMS prior to pregnancy and PPD, independent of history of depression. Evidence to date suggests PMS has the potential to help identify women at increased risk of PPD before pregnancy.

\section{Keywords}

Postpartum depression, premenstrual syndrome, risk factor, screening, early identification, prevention

\subsection{Introduction}

Postpartum depression (PPD) refers to depression that occurs within one year after childbirth (Stewart and Vigod, 2016). It affects about 1 in 6 women who give birth in Australia (Woolhouse et al., 2012). Women who develop PPD are more likely to develop long-term maternal depression (Goodman, 2004; Stewart and Vigod, 2016). PPD may also interfere with breastfeeding, maternalinfant bonding and partner relationships, and impair emotional and cognitive development in children (Viguera, 2018). Given this, early identification of women at increased risk for PPD is of great importance (Stowe and Nemeroff, 1995).

Established risk factors for PPD include a prior history of depression, poor social support, and pregnancy/postpartum complications (Ghaedrahmati et al., 2017). A possible risk factor for PPD - premenstrual syndrome (PMS) - refers to a variety of physical and emotional symptoms that repeatedly occur during the second half of the menstrual cycle (luteal phase) (Casper, 2020). Mood swings, irritability, tension, feeling sad or experiencing depression are the common emotional manifestations of PMS (Yonkers). Due to the fact that oestrogen and progesterone regulate biological systems, neural networks, and behaviours associated with mood, changes in the levels of the two hormones may trigger depressive symptoms (Schiller et al., 2016). As the abrupt drop in hormonal levels take place in the luteal phase as well as after delivery, women who are susceptible to hormonal fluctuations might get both PMS and PPD (Schiller et al., 2016; Yonkers et al., 2008). 
In a recent systematic review and meta-analysis (Cao et al., 2020), we concluded that the evidence overall supported a positive association between history of PMS and development of PPD. A limitation of this review is that many of the included studies were of low-quality, for example they used a cross-sectional or case-control design, which can only detect the co-occurrence of PMS and PPD. Other cohort studies (Limlomwongse and Liabsuetrakul, 2006; Martini et al., 2015; Sylvén et al., 2013; Turkcapar et al., 2015; Wang, 2008; Zhang, 2011) have investigated the association between pre-pregnancy PMS and PPD, but they are limited by convenience sampling, i.e. recruiting participants through the hospital where women delivered their baby, and not distinguishing women who develop PPD as their first depressive episode from those who had a previous history of depression. In view of the potentially different pathophysiology (Altemus et al., 2012; Kettunen et al., 2014) and risk factors (Silverman et al., 2017) between PPD with or without a prior depression, the association between PMS and PPD may be affected by history of depression. However, no studies so far have accounted for this.

Another drawback of previous studies is the investigation of the association between PMS and PPD in only one birth of the participants. A longitudinal cohort that tracks participants over the years can capture the development of PPD in more than one birth as well as the changing status of PMS and other covariates over time. Using such data may assist in a more thorough examination of potential confounders for the association between pre-pregnancy PMS and PPD.

In this study, we used a prospective cohort of women who were followed up for 15 years from 22-27 years to 37-42 years to examine the longitudinal association of PMS before pregnancy with the risk of PPD development. Our hypothesis was that PMS before pregnancy increases the risk of PPD development, and history of depression will affect this association.

\subsection{Material and methods}

\subsubsection{Participants and study design}

The 1973-78 cohort of the Australian Longitudinal Study on Women's Health (ALSWH) formed the study population. In 1996, the ALSWH recruited a nationally representative sample of over 40000 women from the Medicare database in three age cohorts, born in 1921-26, 1946-51 and 1973-78, and these cohorts have been followed at approximate 3-yearly intervals since then via postal and/or online surveys (Lee et al., 2005). The aim of the ALSWH is to examine the social, psychological, physical and environmental factors which determine good health, and those which cause ill health, in women across the life course, with particular emphasis on women's access to and use of health services. Detailed survey methods have been previously published (Dobson et al., 2015). The 1973-78 cohort consists of women who were aged 18-23 years in 1996, and up to 2018 had completed eight surveys. 
For this analysis, data over 15 years from Survey 2 (conducted in 2000 when the women were 22-27 years) to Survey 7 (in 2015, when 37-42 years) were used. Survey 1 was not included since PPD was not collected, and at the time of analysis data from the most recent survey (in 2018) was not yet available. Hence, baseline refers to Survey 2 in this analysis. Figure 1 shows the process of participant selection, with 5479 women and their 10722 births during the 15 years of follow-up included in the analysis. Women included were those who were not pregnant at baseline, had at least one live birth between Survey 2 and Survey 7, and had no previous history of PPD. Prepregnancy characteristics, including PMS and other covariates, were collected from the survey preceding each birth to analyse the association of interest. For example, if a woman had a child between Survey 3 and 4, pre-pregnancy factors collected at Survey 3 were used. If she then had a second birth between Survey 6 and 7, pre-pregnancy factors collected at Survey 6 were used.

\subsubsection{Measurements}

All data used were from the women's self-report.

Exposure

The presence of PMS was reported at each survey using the 4-category Likert-scale: "In the last 12 months, have you had premenstrual tension? (never, rarely, sometimes, or often)".

\section{Outcome}

In each of Surveys 5, 6 and 7, the women were asked to retrospectively record whether they had PPD following the birth of every child, with the question "Were you diagnosed or treated for postnatal depression?" (yes/no). If women did not complete these questions fully for every birth, this missing information was able to be inferred for some women using a question that had been asked in each of Surveys 2, 3 and 4; "In the last 3 or 4 years, have you been told by a doctor that you have postnatal depression?" (yes/no). We were able to use the children's date of birth, together with the date the women completed Surveys 2 to 4 to ascertain whether the women had reported PPD for that particular birth.

\section{Covariates}

The following sociodemographic factors and common risk factors of PMS and PPD were included as covariates, which were repeatedly collected at each survey: age, highest qualification (university degree or higher, high school/trade/diploma and less than high school), marital status (married/de facto and not married/separated/widowed), area of residence (major cities, inner regional and outer regional/remote/very remote), ability to manage on income (easy, not too bad, difficult sometimes and difficult always/impossible), social support (all of the time, most of the time and some/a little/none of the time), body mass index (BMI, $<18.5,18.5$ to $<25,25$ to $<30$ and $\geq 30$ ), physical activity (sedentary, low, moderate and high), smoking (never smoker, ex-smoker and current 
smoker), oral contraceptives (OCPs) use (not using and using), and history of depression (based on either self-report of doctor-diagnosed depression or use of antidepressants both asked at each survey; for both measures women were classified as having a history of depression if they answered yes at any preceding survey).

Age at menarche ( $\leqslant 11,12-14$ and $\geqslant 15$ years) (Harlow et al., 2004; Mendle et al., 2018), parity (primiparous/multiparous) (Di Florio et al., 2014; Iwata et al., 2016), gestational diabetes (yes/no) (Arafa and Dong, 2019; Azami et al., 2019) and preterm birth (yes/no) (de Paula Eduardo et al., 2019; Helle et al., 2015) were also included as covariates since previous literature has reported a positive association with depression or PPD.

\subsubsection{Statistical analysis}

Baseline characteristics of the women and univariable associations with prospective development of PPD were described and compared using descriptive statistics, chi-square, or t-tests. Relative risks (RRs) and 95\% confidence intervals (CIs) were used to report the longitudinal association between PMS, covariates and PPD, calculated from log-binomial or log-Poisson regression (when the logbinomial model did not converge) models with generalised estimating equations (GEE) to account for the correlation between births of the same woman.

PPD was regressed on PMS reported in the survey before the index birth, as well as the other covariates. First, the longitudinal univariable analysis was performed. Significant factors identified from the univariable analysis were included in the multivariable analysis. Statistical significance was set at $P$-value $<0.05$. Although OCPs use was not significantly associated with PPD in the univariable analysis, it was included in the adjusted models because OCPs are commonly used for treating PMS (Mayo Clinic) and have been recently reported as being associated with PPD development (Horibe et al., 2018; Roberts and Hansen, 2017). The fully adjusted models controlled for the following covariates for the index birth: sociodemographic factors (highest qualification, marital status and difficulty on managing income), reproductive factors (maternal age, parity, gestational diabetes and preterm birth), lifestyle factors (pre-pregnancy BMI, physical activity, smoking, and OCPs use), social support, and history of depression.

To examine whether the association between PMS and PPD was affected by history of depression, we tested the interaction between PMS and history of depression by including a product term in the fully adjusted model.

To assess the robustness of the findings, we performed three sensitivity analyses. First, the history of doctor-diagnosed depression was replaced with the history of any prior use of antidepressants. Second, to assess the influence of participants exclusion due to missing values in 
covariates ( $\mathrm{n}=539)$, PROC MI was used to impute missing values in PMS, highest qualification, marital status, ability to manage income, pre-pregnancy BMI, physical activity, smoking, OCP use, and social support. Association between PMS and PPD was assessed in 10 imputed datasets, and the effect estimates were then pooled using PROC MIANALYZE. Third, the study sample was restricted to primiparous women and their first births. All statistical analysis was conducted using SAS 9.4 for Windows (SAS Institute Inc., Cary, NC, USA).

\subsubsection{Ethical approval}

Ethical approvals for the project were granted from the Human Research Ethics Committees of the University of Newcastle (Approval No. H-076-0795) and the Medical Research Ethics Committee of the University of Queensland (Approval No. 2004000224 and 2012000950). All participants gave signed informed consent, and all data used in the analyses were de-identified.

\subsection{Results}

\subsubsection{Baseline characteristics, experience of PMS and development of PPD}

Among the 5479 women who had at least one live birth during the 15 years of follow-up, 842 women (15.4\%) reported having PPD in 887 births, and 3019 women (55.1\%) reported experiencing PMS (rarely: 17.2\%, sometimes: $25.7 \%$, and often: $12.2 \%$ ). PPD was more likely to occur in women with less education, who had more difficulty in managing their income, had less social support, had a history of depression and who were current smokers at baseline (Table 1).

\subsubsection{Association between pre-pregnancy PMS and PPD}

The univariable association between covariates and PPD are shown in Table S1. Table 2 shows a dose-response relationship between pre-pregnancy PMS and PPD after adjustment for all covariates. That is, compared to women who had no PMS before pregnancy, those who rarely had PMS had similar risk of developing PPD (1.03, 0.82-1.30); those who sometimes had PMS had higher risk of PPD (1.31, 1.09-1.57); and those who often had PMS had the highest risk of getting PPD $(1.51,1.22-1.87)$.

\subsubsection{The role of history of depression in the association between PMS and PPD}

No significant interaction was detected between pre-pregnancy PMS and history of depression ( $P$-value for interaction: 0.47$)$.

\subsubsection{Summary of sensitivity analyses}

The results of sensitivity analyses are shown in Table S2, which all support a dose-response relationship between pre-pregnancy PMS and PPD, except for the non-statistically significant 
increased risk of PPD among primiparous women who sometimes had PMS symptoms before pregnancy $(1.15,0.92-1.44)$.

\subsection{Discussion}

In this longitudinal cohort study from Australia, we evaluated the association between PMS and PPD development among 5479 women, who had given birth during the 15 years of follow-up from age 22-27 to 37-42 years. We observed that PMS before pregnancy was positively associated with PPD in a dose-response fashion, and this relationship was independent of the history of depression.

\subsubsection{Interpretation and comparisons with other studies}

By tracking a nationally representative Australian sample from when they were aged in their twenties to early forties, our study was able the capture of occurrences of PMS and PPD as well as time-varying covariates over 15 years. This time period covered almost the full length of the women's childbearing years and included all their births during this period. With the application of GEE models and autoregressive working correlation, we took account of the correlation between repeated births of each woman, as well as the impact of the PPD occurrence on the subsequent births. As a result, the population-averaged effect of pre-pregnancy PMS on PPD development was estimated.

The increased risk for PPD observed among women with pre-pregnancy PMS is consistent with the finding of a meta-analysis that we recently published, in which we reported that women who had PMS before pregnancy had more than double the odds of developing PPD (odds ratio (OR), 95\% CI: 2.20, 1.81-2.68) based on current literature (Cao et al., 2020). Several prospective cohort studies have reported similar findings, even though they have only evaluated the association of interest in one birth per woman and dichotomised the status of PMS into having or not having (Limlomwongse and Liabsuetrakul, 2006; Sylvén et al., 2013; Turkcapar et al., 2015; Wang, 2008; Zhang, 2011). For example, a Turkish cohort study including 540 women observed that women with a history of PMS had twice the odds of PPD compared to those who had no PMS before pregnancy (OR: 2.05, 1.25-2.33) (Turkcapar et al., 2015). A Swedish study led by Sylvén et al. measured PPD in 1308 women three times after delivery and found that history of PMS was associated with PPD at five days and six weeks after birth (Sylvén et al., 2013).

The finding that the relationship between PMS and PPD was independent of history of depression did not support our hypothesis. This is slightly different to the finding of another study led by Sylvén and colleagues (Sylvén et al., 2017), in which they concluded that PMS before pregnancy was associated with PPD at five days (crude OR: 3.5, 1.2-10.0) but not at six weeks 
postpartum (crude OR:0.98, 0.1-7.7) in 653 first-time mothers without any previous contact with psychiatric services.

The underlying biological mechanism for the association of PMS and PPD may relate to the role of oestrogen and progesterone in mood regulation. Oestrogen and progesterone are involved in regulating biological systems, neural networks, and behaviours associated with mood; therefore, changes in the level of the two hormones may trigger affective dysregulation and lead to depressive symptoms (Schiller et al., 2016). Because the levels of both hormones abruptly drop during the luteal phase as well as after delivery, women who are sensitive to hormonal fluctuations could experience both PMS and PPD (Schiller et al., 2016; Yonkers et al., 2008). However, for women who had a history of PMS but developed PPD in the late postpartum period (the level of hormones remains stable at this point), this explanation may not apply. Psychosocial factors, such as external stressors may also play a role in the link between pre-pregnancy PMS and PPD development (Halbreich, 2005). Future investigations of the theoretical underpinnings of the positive association between PMS and PPD are warranted.

\subsubsection{Strengths and limitations}

A unique strength of this present study is the longitudinal study design. This is, to our knowledge, the first study to use a longitudinal cohort to investigate the prospective association between PMS before pregnancy and development of PPD. As a population-based study, the exposure and most covariates were asked at each survey, regardless of whether women were planning or not to get pregnant, which to a large extent prevented the selection bias of participants. Besides, a comprehensive adjustment for confounders that may have affected the association of interest, such as maternal age at birth, parity, pregnancy complications, social support, and history of depression, was conducted. In addition, sensitivity analyses, which generated similar results, indicated the robustness of our findings. Furthermore, the finding that the association of interest was independent of women's previous history of depression highlighted the biological and/or psychosocial link between PMS and PPD. This has implications for the early identification of women at risk of PPD based on their history of PMS. Finally, since participants in this study were largely nationally representative, our results can be generalised to Australian women of the same age.

Limitations of the present study included at least three aspects. First, for the main analysis, identification of PPD was ascertained from women's self-reports of doctor-diagnosed or treated PPD for each birth, which might be slightly different from the current definition of PPD specified in the Diagnostic and Statistical Manual of Mental Disorders Fifth Edition (DSM-V) (American Psychiatric Association, 2013). Introduced in 2013, the DSM-V required the onset of depressive 
symptoms to occur during pregnancy or within 4 weeks following delivery. Participants in our study who had given birth before 2013 (which equated to Surveys 2 to 6) could have been diagnosed for PPD with different criteria, especially those who had a late onset of depressive symptoms after delivery. However, multiple administrative data sources have shown the accuracy of our self-reported PPD measures. We previously found that $63 \%$ of our self-reported PPD cases had received antidepressants treatment or Medicare-subsidised mental health services in the first postpartum year, and $72-75 \%$ of the diagnosed cases retrieved from hospital records were correctly reported by women in the survey (data on request). The association observed in our study might have underestimated the true relationship between pre-pregnancy PMS and PPD risk if underreporting of PPD existed. In an additional analysis (Table S3), we used the women's scores on the Mental Health Subscale from the SF-36 within one year of delivery to infer PPD symptoms, instead of the doctor diagnosed PPD. Using survival analysis, which accounted for the time from birth to when women completed the subscale, we observed a 75\% higher risk of PPD for women who often experienced PMS before pregnancy (Hazard Ratio: 1.75, 1.19-2.57). This to some extent strengthened our findings.

Second, the experience of PMS was derived from the question asking about premenstrual tension retrospectively, which may result in recall bias. Premenstrual tension is the old term defined in the International Statistical Classification of Diseases and Related Health Problems, Tenth Edition (ICD-10) describing premenstrual symptoms such as tension or migraine without consideration of the type or severity of the symptoms (Pearlstein, 2007). This is broader than the definition of core premenstrual disorders specified in recent PMS consensus/guidelines (Nevatte et al., 2013; Royal College of Obstetricians \& Gynaecologists, 2017; World Health Organization, 2018). Although we categorised women into four levels of PMS frequency, the severity of PMS was not measured. Third, due to the 3-year interval between two consecutive surveys, PMS history for some women might have been up to 3 years before the index pregnancy, but on average, the PMS history was 0.8 year before the index pregnancy.

\subsubsection{Clinical implications}

Both the Australian (Centre of Perinatal Excellence, 2017) and the US (US Preventive Services Task Force, 2019) perinatal mental health guidelines recommend screening for psychosocial risk factors of PPD during pregnancy and after delivery to identify women at risk; however, very limited factors have been included in these guidelines, including previous history of depression, lack of social support and low socioeconomic status. Findings from the present study highlight the potential of PMS to identify women at risk of PPD well before they fall pregnant. If the results were to be replicated in different populations, a new PPD screening list might be developed in future, 
incorporating PMS history in addition to those socioeconomic and psychiatric factors that are currently used to screen women at increased risk of PPD during or after pregnancy (Centre of Perinatal Excellence, 2017; US Preventive Services Task Force, 2019).

\subsection{Conclusions}

A dose-response relationship was observed between pre-pregnancy PMS and PPD risk in the ALSWH 1973-78 cohort, and the association was independent of the history of depression. The findings highlight that PMS has the potential to identify women at risk of PPD well before their pregnancy.

\section{Acknowledgements}

The research on which this paper is based was conducted as part of the Australian Longitudinal Study on Women's Health by the University of Queensland and the University of Newcastle. We are grateful to the Australian Government Department of Health for funding and to the women who provided the survey data. 


\section{References}

Altemus, M., Neeb, C.C., Davis, A., Occhiogrosso, M., Nguyen, T., Bleiberg, K.L., 2012. Phenotypic differences between pregnancy-onset and postpartum-onset major depressive disorder. J. Clin. Psychiatry. 73, e1485-1491.

American Psychiatric Association, 2013. Diagnostic and statistical manual of mental disorders, 5th ed: DSM-V. American Psychiatric Association Publising, Washington, DC.

Arafa, A., Dong, J.Y., 2019. Gestational diabetes and risk of postpartum depressive symptoms: A meta-analysis of cohort studies. J. Affect. Disord. 253, 312-316.

Azami, M., Badfar, G., Soleymani, A., Rahmati, S., 2019. The association between gestational diabetes and postpartum depression: A systematic review and meta-analysis. Diabetes Res. Clin. Pract. 149, 147-155.

Cao, S., Jones, M., Tooth, L., Mishra, G.D., 2020. History of premenstrual syndrome and development of postpartum depression: A systematic review and meta-analysis. J. Psychiatr. Res. $121,82-90$.

Casper, R.F., 2020. Patient education: Premenstrual syndrome (PMS) and premenstrual dysphoric disorder (PMDD) (Beyond the Basics). https://www.uptodate.com/contents/premenstrualsyndrome-pms-and-premenstrual-dysphoric-disorder-pmdd-beyond-the-basics (accessed 23 April 2020).

Centre of Perinatal Excellence, 2017. Mental Health Care in the Perinatal Period: Australian Clinical Practice Guideline. https://www.cope.org.au/wp-content/uploads/2018/05/COPE-Perinatal-MHGuideline Final-2018.pdf (accessed 20 March 2018).

de Paula Eduardo, J.A.F., de Rezende, M.G., Menezes, P.R., Del-Ben, C.M., 2019. Preterm birth as a risk factor for postpartum depression: A systematic review and meta-analysis. J. Affect. Disord. 259, 392-403.

Di Florio, A., Jones, L., Forty, L., Gordon-Smith, K., Blackmore, E.R., Heron, J., Craddock, N., Jones, I., 2014. Mood disorders and parity - a clue to the aetiology of the postpartum trigger. J. Affect. Disord. 152-154, 334-339.

Dobson, A.J., Hockey, R., Brown, W.J., Byles, J.E., Loxton, D.J., McLaughlin, D., Tooth, L.R., Mishra, G.D., 2015. Cohort Profile Update: Australian Longitudinal Study on Women's Health. Int. J. Epidemiol. 44, 1547,1547a-1547f.

Ghaedrahmati, M., Kazemi, A., Kheirabadi, G., Ebrahimi, A., Bahrami, M., 2017. Postpartum depression risk factors: A narrative review. J. Educ. Health. Promot. 6, 60.

Goodman, J.H., 2004. Postpartum depression beyond the early postpartum period. J. Obstet. Gynecol. Neonatal Nurs. 33, 410-420.

Halbreich, U., 2005. Postpartum disorders: Multiple interacting underlying mechanisms and risk factors. J. Affect. Disord. 88, 1-7.

Harlow, B.L., Cohen, L.S., Otto, M.W., Spiegelman, D., Cramer, D.W., 2004. Early life menstrual characteristics and pregnancy experiences among women with and without major depression: the Harvard study of moods and cycles. J. Affect. Disord. 79, 167-176.

Helle, N., Barkmann, C., Bartz-Seel, J., Diehl, T., Ehrhardt, S., Hendel, A., Nestoriuc, Y., SchulteMarkwort, M., von der Wense, A., Bindt, C., 2015. Very low birth-weight as a risk factor for postpartum depression four to six weeks postbirth in mothers and fathers: Cross-sectional results from a controlled multicentre cohort study. J. Affect. Disord. 180, 154-161. 
Horibe, M., Hane, Y., Abe, J., Matsui, T., Kato, Y., Ueda, N., Sasaoka, S., Motooka, Y., Hatahira, H., Hasegawa, S., Kinosada, Y., Hara, H., Nakamura, M., 2018. Contraceptives as possible risk factors for postpartum depression: A retrospective study of the food and drug administration adverse event reporting system, 2004-2015. Nurs. Open. 5, 131-138.

Iwata, H., Mori, E., Sakajo, A., Aoki, K., Maehara, K., Tamakoshi, K., 2016. Prevalence of postpartum depressive symptoms during the first 6 months postpartum: Association with maternal age and parity. J. Affect. Disord. 203, 227-232.

Kettunen, P., Koistinen, E., Hintikka, J., 2014. Is postpartum depression a homogenous disorder: time of onset, severity, symptoms and hopelessness in relation to the course of depression. BMC Pregnancy Childbirth. 14, 402.

Lee, C., Dobson, A.J., Brown, W.J., Bryson, L., Byles, J., Warner-Smith, P., Young, A.F., 2005. Cohort profile: the Australian longitudinal study on women's health. Int. J. Epidemiol. 34, 987-991.

Limlomwongse, N., Liabsuetrakul, T., 2006. Cohort study of depressive moods in Thai women during late pregnancy and 6-8 weeks of postpartum using the Edinburgh Postnatal Depression Scale (EPDS). Arch. Women's Ment. Health. 9, 131-138.

Martini, J., Petzoldt, J., Einsle, F., Beesdo-Baum, K., Höfler, M., Wittchen, H.U., 2015. Risk factors and course patterns of anxiety and depressive disorders during pregnancy and after delivery: $\mathrm{A}$ prospective-longitudinal study. J. Affect. Disord. 175, 385-395.

Mayo Clinic, Premenstrual syndrome (PMS) - Diagnosis and treatment. https://www.mayoclinic.org/diseases-conditions/premenstrual-syndrome/diagnosistreatment/drc-20376787 (accessed March, 9 2020).

Mendle, J., Ryan, R.M., McKone, K.M.P., 2018. Age at Menarche, Depression, and Antisocial Behavior in Adulthood. Pediatrics. 141, e20171703.

Nevatte, T., O'Brien, P.M., Backstrom, T., Brown, C., Dennerstein, L., Endicott, J., Epperson, C.N., Eriksson, E., Freeman, E.W., Halbreich, U., Ismail, K., Panay, N., Pearlstein, T., Rapkin, A., Reid, R., Rubinow, D., Schmidt, P., Steiner, M., Studd, J., Sundstrom-Poromaa, I., Yonkers, K., 2013. ISPMD consensus on the management of premenstrual disorders. Arch. Women's Ment. Health. 16, 279291.

Pearlstein, T., 2007. Prevalence, impact on morbidity, and disease burden, In: P. M. Shaughn O'Brien, A.R., Peter J. Schmidt (Ed.), The Premenstrual Syndromes: PMS and PMDD, 1st ed. CRC Press, London, pp. 10-11.

Roberts, T.A., Hansen, S., 2017. Association of Hormonal Contraception with depression in the postpartum period. Contraception. 96, 446-452.

Royal College of Obstetricians \& Gynaecologists, 2017. Management of Premenstrual Syndrome: Green-top Guideline No. 48. BJOG. 124, e73-e105.

Schiller, C.E., Johnson, S.L., Abate, A.C., Schmidt, P.J., Rubinow, D.R., 2016. Reproductive Steroid Regulation of Mood and Behavior. Compr. Physiol. 6, 1135-1160.

Silverman, M.E., Reichenberg, A., Savitz, D.A., Cnattingius, S., Lichtenstein, P., Hultman, C.M., Larsson, H., Sandin, S., 2017. The risk factors for postpartum depression: A population-based study. Depress Anxiety. 34, 178-187.

Stewart, D.E., Vigod, S., 2016. Postpartum depression. N Engl J Med. 375, 2177-2186.

Stowe, Z.N., Nemeroff, C.B., 1995. Women at Risk for Postpartum-Onset Major Depression. Am. J. Obstet. Gynecol. 173, 639-645. 
Sylvén, S.M., Ekselius, L., Sundström-Poromaa, I., Skalkidou, A., 2013. Premenstrual syndrome and dysphoric disorder as risk factors for postpartum depression. Acta Obstet. Gynecol. Scand. 92, 178-184.

Sylvén, S.M., Thomopoulos, T.P., Kollia, N., Jonsson, M., Skalkidou, A., 2017. Correlates of postpartum depression in first time mothers without previous psychiatric contact. Eur. Psychiatry. 40, 4-12.

Turkcapar, A.F., Kadioglu, N., Aslan, E., Tunc, S., Zayifoglu, M., Mollamahmutoglu, L., 2015. Sociodemographic and clinical features of postpartum depression among Turkish women: a prospective study. BMC Pregnancy Childbirth. 15, 108.

US Preventive Services Task Force, 2019. Interventions to Prevent Perinatal Depression: US Preventive Services Task Force Recommendation Statement. JAMA. 321, 580-587.

Viguera, A., 2018. Postpartum unipolar major depression: Epidemiology, clinical features, assessment, and diagnosis. https://www.uptodate.com/contents/postpartum-unipolar-majordepression-epidemiology-clinical-features-assessment-and-diagnosis\#H4004681033 (accessed 28 June 2018).

Wang, R., 2008. Study of related psychosocial factors for postpartum depression. China Medical University.

Woolhouse, H., Gartland, D., Hegarty, K., Donath, S., Brown, S.J., 2012. Depressive symptoms and intimate partner violence in the 12 months after childbirth: a prospective pregnancy cohort study. BJOG : an international journal of obstetrics and gynaecology. 119, 315-323.

World Health Organization, 2018. International Classification of Diseases, 11th Revision (ICD-11). https://icd.who.int/browse11/l-m/en\#http\%3a\%2f\%2fid.who.int\%2ficd\%2fentity\%2f375471908 (accessed 30 October 2019).

Yonkers, K.A., O'Brien, P.M., Eriksson, E., 2008. Premenstrual syndrome. Lancet. 371, 1200-1210.

Yonkers, K.C., M. F., Clinical manifestations and diagnosis of premenstrual syndrome and premenstrual dysphoric disorder. https://www.uptodate.com/contents/clinical-manifestationsand-diagnosis-of-premenstrual-syndrome-and-premenstrual-dysphoric-disorder (accessed).

Zhang, Y., 2011. Study of psychosocial factors for postpartum depression among 479 women. Huazhong University of Science and Technology. 


\section{Figure Caption List}

Figure 1. Flowchart of participant selection

\section{Table list}

Table 1. Baseline characteristics of non-pregnant women according to prospective development of $\operatorname{PPD}(\mathrm{n}=5479)$

Table 2. Unadjusted and adjusted relative risks and 95\% confidence intervals for the association of pre-pregnancy PMS and PPD (n=4586)

Table S1. Univariable analysis of the association between possible risk factors and PPD $(n=5479)$

Table S2. Summary of the association between pre-pregnancy PMS and PPD in sensitivity analyses

Table S3. Association between pre-pregnancy PMS and PPD symptoms $(\mathrm{n}=2375)$ 


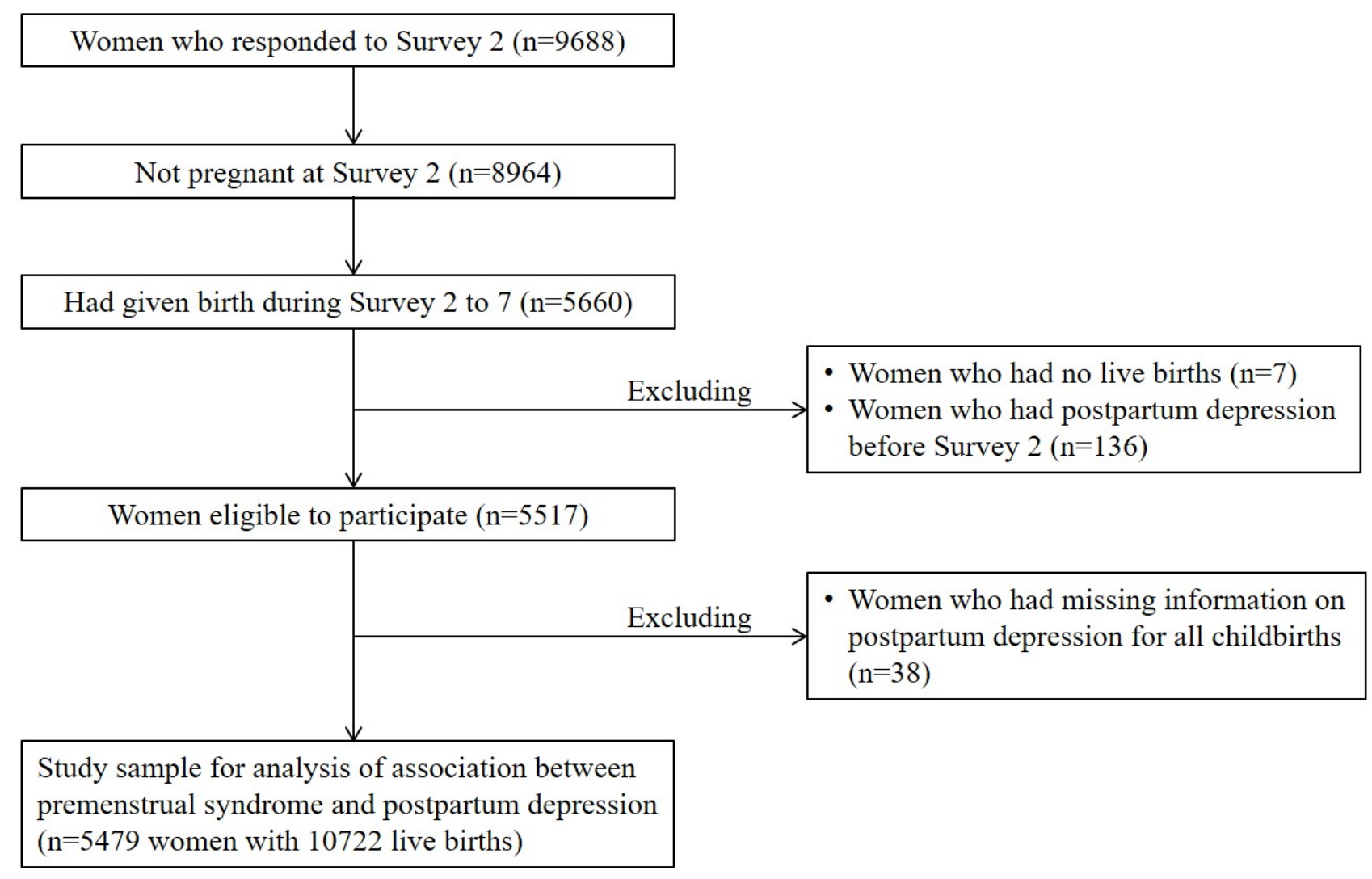

Figure 1. Flowchart of participant selection 
Table 1. Baseline characteristics of non-pregnant women according to prospective development of $\operatorname{PPD}(\mathrm{n}=5479)^{\mathrm{a}}$

\begin{tabular}{|c|c|c|c|c|}
\hline & $\begin{array}{c}\text { No PPD, } \\
\mathrm{n}=4637 \\
\mathrm{n}(\%)\end{array}$ & $\begin{array}{c}\mathrm{PPD}, \\
\mathrm{n}=842 \\
\mathrm{n}(\%)\end{array}$ & $\chi^{2}$ & P-value \\
\hline Years of age, $n=5479^{b}$ & $24.1 \pm 1.4$ & $24 \pm 1.5$ & - & 0.34 \\
\hline Highest qualification, $n=5303$ & & & 6.47 & 0.04 \\
\hline University degree or higher & $2087(46.5)$ & $342(42.0)$ & & \\
\hline High school/trade/diploma & $2047(45.6)$ & $410(50.4)$ & & \\
\hline Less than high school & $355(7.9)$ & $62(7.6)$ & & \\
\hline Marital status, $\mathrm{n}=5456$ & & & 0.21 & 0.64 \\
\hline Married/de facto & $2247(48.7)$ & $415(49.5)$ & & \\
\hline Not married/separated/widowed & $2371(51.3)$ & $423(50.5)$ & & \\
\hline \multicolumn{5}{|l|}{ Area of residence, $n=5454$} \\
\hline Major cities & $2421(52.4)$ & $452(54.0)$ & 1.63 & 0.44 \\
\hline Inner regional & $1392(30.2)$ & $234(28.0)$ & & \\
\hline Outer regional/remote/very remote & $804(17.4)$ & $151(18.0)$ & & \\
\hline $\begin{array}{l}\text { Ability to manage on income, } \\
n=4804\end{array}$ & & & 19.99 & $<0.001$ \\
\hline Easy & $507(12.5)$ & 73 (9.9) & & \\
\hline Not too bad & $1933(47.6)$ & $312(42.1)$ & & \\
\hline Difficult sometimes & $1305(32.1)$ & $274(37.0)$ & & \\
\hline Difficult always/impossible & $318(7.8)$ & $82(11.1)$ & & \\
\hline Social support, $\mathrm{n}=5391$ & & & 9.03 & 0.01 \\
\hline All of the time & $2818(61.8)$ & $467(56.3)$ & & \\
\hline Most of the time & $1196(26.2)$ & $243(29.3)$ & & \\
\hline Some/a little/none of the time & $548(12.1)$ & $119(14.4)$ & & \\
\hline Age at menarche, $n=5444$ & & & 1.87 & 0.39 \\
\hline $8-11$ years & $544(11.8)$ & $111(13.3)$ & & \\
\hline 12-14 years & $3504(76.1)$ & $620(74.0)$ & & \\
\hline$\geq 15$ years & $558(12.1)$ & $107(12.8)$ & & \\
\hline BMI, $\mathrm{n}=5363$ & & & 5.72 & 0.13 \\
\hline Underweight $(<18.5)$ & $277(6.1)$ & $44(5.4)$ & & \\
\hline Normal weight $(18.5$ to $<25)$ & $3039(66.9)$ & $526(63.9)$ & & \\
\hline Overweight $(25$ to $<30)$ & $858(18.9)$ & $172(20.9)$ & & \\
\hline Obese $(\geq 30)$ & $366(8.1)$ & $81(9.8)$ & & \\
\hline Physical activity, $\mathrm{n}=5357$ & & & 4.55 & 0.21 \\
\hline Sedentary & $361(8.0)$ & $80(9.7)$ & & \\
\hline Low & $1375(30.3)$ & $260(31.6)$ & & \\
\hline
\end{tabular}


Moderate

High

Smoking, $\mathrm{n}=5444$

Never smoker

Ex-smoker

Current smoker

OCPs use, $n=5441$

Not using OCPs

Using OCPs

History of depression, $\mathrm{n}=5479$

No

Yes
1128 (24.9)

$1671(36.9)$

$202(24.6)$

$280(34.1)$

(60.6)

$647(14.0)$

$1169(25.4)$

$1642(35.6)$

2967 (64.4)

(9.6)

$437(9.4)$
$19.19<0.001$

$446(53.5)$

118 (14.2)

$270(32.4)$

$0.09 \quad 0.76$

301 (36.2)

$531(63.8)$

$167.05<0.001$

$631(74.9)$

$211(25.1)$

a Percentages may not add up to $100 \%$ due to rounding.

${ }^{\mathrm{b}}$ Mean $\pm \mathrm{SD}$ 
Table 2. Unadjusted and adjusted relative risks and 95\% confidence intervals for the association of pre-pregnancy PMS and PPD (n=4586)

\begin{tabular}{lccccc}
\hline PMS & Crude RR & Model 1 & Model 2 & Model 3 $^{\mathrm{b}}$ & Model 4 $^{\mathrm{d}}$ \\
\hline Never & Reference & Reference & Reference & Reference & Reference \\
Rarely & $1.05(0.84-1.32)$ & $1.05(0.84-1.31)$ & $1.01(0.80-1.27)$ & $1.01(0.80-1.27)$ & $1.03(0.82-1.30)$ \\
Sometimes & $1.52(1.27-1.83)$ & $1.50(1.25-1.80)$ & $1.36(1.13-1.64)$ & $1.36(1.13-1.64)$ & $1.31(1.09-1.57)$ \\
Often & $2.20(1.77-2.74)$ & $2.10(1.68-2.62)$ & $1.74(1.39-2.17)$ & $1.71(1.37-2.13)$ & $1.51(1.22-1.87)$ \\
\hline
\end{tabular}

${ }^{a}$ Model 1 adjusted for highest qualification, marital status, and ability to manage income

${ }^{\mathrm{b}}$ Model 2 adjusted for variables in Model 1 plus for maternal age, parity, gestational diabetes, preterm birth, pre-pregnancy BMI, physical activity, smoking and OCP use

${ }^{c}$ Model 3 adjusted for variables in Model 2 plus for social support

${ }^{\mathrm{d}}$ Model 4 adjusted for variables in Model 3 plus for history of depression 
Table S1. Univariable analysis of the association between possible risk factors and PPD $(n=5479)$

\begin{tabular}{|c|c|c|}
\hline Variables & $\mathrm{RR}(95 \% \mathrm{CI}) *$ & $\mathrm{P}$ value \\
\hline Maternal age (continuous) & $0.94(0.93-0.96)$ & $<0.001$ \\
\hline Highest qualification & & $<0.001$ \\
\hline University degree or higher & Reference & \\
\hline High school/trade/diploma & $1.44(1.25-1.66)$ & \\
\hline Less than high school & $1.17(0.88-1.56)$ & \\
\hline Marital status & & $<0.001$ \\
\hline Married/de facto & Reference & \\
\hline Not married/separated/widowed & $1.56(1.28-1.88)$ & \\
\hline Area of residence & & 0.94 \\
\hline Major cities & Reference & \\
\hline Inner regional & $1.02(0.86-1.20)$ & \\
\hline Outer regional/remote/very remote & $0.98(0.81-1.18)$ & \\
\hline Ability to manage on income & & $<0.001$ \\
\hline Easy & Reference & \\
\hline Not too bad & $1.02(0.85-1.22)$ & \\
\hline Difficult sometimes & $1.30(1.07-1.57)$ & \\
\hline Difficult always/impossible & $1.44(1.12-1.86)$ & \\
\hline Social support & & 0.01 \\
\hline All of the time & Reference & \\
\hline Most of the time & $1.05(0.90-1.23)$ & \\
\hline Some/a little/none of the time & $1.41(1.13-1.75)$ & \\
\hline Age at menarche & & 0.24 \\
\hline $8-11$ years & $1.17(0.96-1.41)$ & \\
\hline $12-14$ years & Reference & \\
\hline$\geq 15$ years & $1.09(0.89-1.33)$ & \\
\hline Premenstrual syndrome & & $<0.001$ \\
\hline Never & Reference & \\
\hline Rarely & $0.97(0.79-1.19)$ & \\
\hline Sometimes & $1.34(1.14-1.59)$ & \\
\hline Often & $2.07(1.71-2.52)$ & \\
\hline Parity & & $<0.001$ \\
\hline Primiparous & Reference & \\
\hline Multiparous & $1.94(1.71-2.20)$ & \\
\hline Gestational diabetes & & $<0.001$ \\
\hline No & Reference & \\
\hline Yes & $1.57(1.21-2.04)$ & \\
\hline Premature birth & & $<0.001$ \\
\hline
\end{tabular}


No

Reference

Yes

$1.73(1.40-2.13)$

Body mass index (BMI)

Underweight $(<18.5)$

Normal weight $(18.5$ to $<25)$

$0.97(0.65-1.44)$

Reference

Overweight $(25$ to $<30)$

$1.26(1.07-1.49)$

Obese $(\geq 30)$

$1.29(1.06-1.57)$

Physical activity

0.04

Sedentary

Reference

Low

$0.78(0.63-0.97)$

Moderate

$0.80(0.64-1.01)$

High

$0.94(0.75-1.18)$

Smoking

$<0.001$

Never smoker

Reference

Ex-smoker

1.25 (1.06-1.47)

Current smoker

$1.58(1.33-1.89)$

OCPs use

Not using OCPs

Reference

Using OCPs

$1.12(0.97-1.28)$

History of depression (doctor-diagnosed)

No

Reference

Yes

$2.49(2.18-2.84)$

History of depression (treated with antidepressants)

No

Reference

$<0.001$

Yes

$2.04(1.69-2.45)$

* Relative risk (95\% confidence interval) 
Table S2. Summary of the association between pre-pregnancy PMS and PPD in sensitivity analyses ${ }^{\text {a }}$

\begin{tabular}{lccc}
\hline \multirow{2}{*}{ PMS } & $\begin{array}{c}\text { Ever use of antidepressants inferring } \\
\text { history of depression }\end{array}$ & $\begin{array}{c}\text { Multiple imputation for missing } \\
\text { data }\end{array}$ & $\begin{array}{c}\text { Inclusion of only primiparous women } \\
\text { and their first births }\end{array}$ \\
\cline { 2 - 4 } Never & Adjusted RR* $(\mathrm{n}=4586)$ & Adjusted RR* $(\mathrm{n}=5125)^{\text {Adjusted RR }^{*}(\mathrm{n}=3601)}$ \\
Rarely & Reference & Reference & Reference \\
Sometimes & $1.02(0.81-1.28)$ & $1.02(0.83-1.26)$ & $1.01(0.76-1.33)$ \\
Often & $1.33(1.10-1.60)$ & $1.28(1.08-1.51)$ & $1.15(0.92-1.44)$ \\
\end{tabular}

${ }^{a}$ Fully adjusted model, adjusting for highest qualification, marital status, ability to manage income, maternal age, parity, gestational diabetes, preterm birth, pre-pregnancy BMI, physical activity, smoking, OCP use, social support, and history of depression

${ }^{\mathrm{b}}$ Missing data was imputed for PMS, highest qualification, marital status, ability to manage income, pre-pregnancy BMI, physical activity, smoking, OCP use, and social support.

* Relative Risk (95\% Confidence Interval), calculated from Log-binomial model with generalized estimating equation

Table S3. Association between pre-pregnancy PMS and PPD symptoms ${ }^{\mathrm{a}}\left(\mathrm{n}=2375^{\mathrm{b}}\right)$

\begin{tabular}{ll}
\hline PMS & Adjusted Hazard Ratio $*$ \\
\hline Never & Reference \\
Rarely & $1.17(0.79-1.76)$ \\
Sometimes & $0.89(0.60-1.32)$ \\
Often & $1.75(1.19-2.57)$ \\
\hline
\end{tabular}

${ }^{a}$ Score of Mental Health Subscale in SF-36 $\leq 52$ in the first postpartum year denoting PPD symptoms

${ }^{\mathrm{b}}$ Only women who filled out the Mental Health Subscale in the first postpartum year were included in analysis

* Calculated from Cox proportional hazards model, which considered the time of the PPD symptoms since delivery, adjusting for highest qualification, marital status, ability to manage income, maternal age, parity, gestational diabetes, preterm birth, pre-pregnancy BMI, physical activity, smoking, OCP use, social support, and history of depression 


\section{RESPONSE TO EDITOR AND REVIEWER}

Aug 20, 2020

Prof. Jair Soares,

Editor-in-Chief,

Journal of Affective Disorders

Dear Professor Soares,

Thank you very much for the opportunity to revise our manuscript. We appreciate the comments and suggestions made by the editor and the reviewers. Please find below a point-by-point response to all comments and a separate revised manuscript attached with changes highlighted.

We hope the revised manuscript is now suitable for publication and look forward to hearing from you again.

Sincerely,

Sifan Cao

Corresponding author, on behalf of all co-authors

The University of Queensland

Faculty of Medicine, School of Public Health, Centre for Longitudinal and Life Course Research

Brisbane, Queensland, Australia

Email: sifan.cao@uq.edu.au 


\section{Comments from Editor}

"I have completed my evaluation of your manuscript. The reviewers recommend reconsideration of your manuscript following revision and modification. I invite you to resubmit your manuscript after addressing the comments below. Please resubmit your revised manuscript by Aug 28, 2020.

When revising your manuscript, please consider all issues mentioned in the reviewers' comments carefully: please outline every change made in response to their comments and provide suitable rebuttals for any comments not addressed. Please note that your revised submission may need to be re-reviewed.

To submit your revised manuscript, please log in as an author at https://www.editorialmanager.com/jafd/, and navigate to the "Submissions Needing Revision" folder under the Author Main Menu.

Journal of Affective Disorders values your contribution and I look forward to receiving your revised manuscript."

\section{$\underline{\text { Response }}$}

Thank you for the opportunity to revise our manuscript. We have undertaken a careful revision of the manuscript according to all comments received.

\section{Comments from Reviewer \# 1}

"This is a study with very large sample size. However, there are some issues to be addressed in the manuscript to make it looks more complete."

\section{$\underline{\text { Response }}$}

All questions raised have been addressed below and corresponding changes are highlighted in the main text.

"Abstract -- The authors should provide the mean age of the subjects and define the definition of "rarely", "sometimes", and "often" since these are the major outcomes of this study."

\section{$\underline{\text { Response }}$}

The age ranges at baseline and the last wave of the 15-year follow-up are now given in the Abstract. "Never", "rarely", "sometimes", and "often" are the pre-defined answers for a 4-category Likertscale reporting PMS experience in the last 12 months. These have been added to the Abstract (lines 9-12).

"Methods -- The authors should clarify the diagnostic validity of the question "In the last 12 months, have you had premenstrual tension?" to diagnose PMS (Is PMT the same as PMS?... or Did all the subjects had been told about the definition of PMT/PMS before asking this question?)"

\section{$\underline{\text { Response }}$}


We have reworded the description of how PMS was collected for clarity (Methods: pg. 3, lines 2-3). As no explanation was provided to participants about the definition of premenstrual tension, no further information was added regarding this in the text.

The question asked in all ALSWH surveys between 2000 and 2015 was "In the last 12 months, have you had premenstrual tension?" "Premenstrual tension", or "premenstrual tension syndrome", is the term for PMS included in the International Classification of Diseases 10th revision (ICD-10) ${ }^{1}$, which acknowledges the existence of the entity of PMS but provides no systematic criteria for diagnosis ${ }^{2}$. A consensus on the diagnosis and classification for PMS was not incorporated in the Royal College of Obstetrics and Gynaecology (RCOG) PMS guideline until $2017^{3}$. Until this time there was a lack of universal agreement on the nature of PMS as well as a lack of universal acceptance of the criteria ${ }^{4}$. We have previously published using the PMS measure ${ }^{5-7}$.

"As well as diagnosis of PPD --> How does the diagnostic validity of the questions "Were you diagnosed or treated for postnatal depression?" (yes/no). "In the last 3 or 4 years, have you been told by a doctor that you have postnatal depression?" (yes/no). Is PPD diagnosed by a psychiatrist, and how to differentiate from postpartum blues? --> The authors may explain in the context of postnatal service in Australia (e.g. every postpartum woman has to visit with a psychiatrist etc.)"

\section{Response}

In this paper, we have used administrative databases such as hospital admission records, Pharmaceutical Benefits Scheme prescriptions, and GP services to assess the accuracy of the selfreported PPD (Discussion: pg. 7 lines 23-27). In addition, we also used postpartum depressive symptoms (yes/no) ascertained from the Mental Health Subscale of SF-36 as another measure for PPD in a sensitivity analysis (Discussion: pg.7 lines 28-33), which also generated similar results (see Table S3).

\section{Comments from Reviewer \# 2}

"This is a large sample study of the association of premenstrual syndrome (PMS) and postpartum depression (PPD). There are no diagnostic criteria both for PMS and PPD in the study, only according to the question" in the last 12 months have you had premenstrual tension?" for PMS, and using antidepressants after delivery for PPD. I think the diagnoses of PMS and PPD in this study are dubious and indeterminate. So the results of the study are uncertain, it will largely interfere the quality of the study. Therefore I consider that this manuscript is not suitable for the journal to publish and recommend the author to send the manuscript to another journal."

\section{$\underline{\text { Response }}$}

Please see our responses to questions from Reviewer \#1. 


\section{References}

1. World Health Organization. International Classification of Diseases, 10th Revision (ICD-10), 2019 Edition. World Health Organization. https://icd.who.int/browse10/2019/en. Published 2019. Accessed 20 August, 2020.

2. Freeman EW. PMS and diagnostic criteria. In: P. M. Shaughn O'Brien AR, Peter J. Schmidt, ed. The Premenstrual Syndromes: PMS and PMDD. 1st ed. London: CRC Press; 2007:9-20.

3. Royal College of Obstetricians \& Gynaecologists. Management of Premenstrual Syndrome: Green-top Guideline No. 48. BJOG. 2017;124(3):e73-e105.

4. Halbreich U. The diagnosis of PMS/PMDD - the current debate. In: P. M. Shaughn O'Brien AR, Peter J. Schmidt, ed. The Premenstrual Syndromes: PMS and PMDD. 1st ed. London: CRC Press; 2007:9-20.

5. Ju H, Jones M, Mishra GD. Premenstrual syndrome and dysmenorrhea: symptom trajectories over 13 years in young adults. Maturitas. 2014;78(2):99-105.

6. Ju H, Jones $M$, Mishra GD. Illicit drug use, early age at first use and risk of premenstrual syndrome: A longitudinal study. Drug Alcohol Depend. 2015;152:209-217.

7. Ju H. The magnitude, long-term trend and lifestyle risk factors of dysmenorrhea and premenstrual syndrome [PhD Thesis]: School of Public Health, The University of Queensland; 2016. 